Finisterra, XXXIII, 65, 1998, pp. 109-116

\title{
QUELQUES RÉFLEXIONS SUR L'INTRODUCTION ET LA VULGARISATION DU CONCEPT DE PAYS AU CAMEROUN
}

ATHANASE BOPDA ${ }^{1}$

Résumé: Parmi les concepts dont l'usage indique l'existence d'une lecture territoriale de l'espace, il en est deux qui reviennent très régulièrement en français: l'Etat et le pays. Nous partons du postulat de base que l'Etat est un territoire de rattachement, à la différence du pays qui est plutôt un territoire d'attachement. Arrivé de la métropole avec un sens plus proche de celui d'Etat, le concept de pays a connu une réinterprétation dans son utilisation par les Camerounais selon les contextes successifs de colonisation, de décolonisation, d'indépendance et, aujourd'hui, de démocratisation.

Mots-clés: Colonisation, décolonisation, pays, Etat, Cameroun.

\begin{abstract}
Some RefleXions on the MeAning of THE ConcePtS OF STATE AND "PAys" IN CAMEROON - In the French language there are two concepts very much used for the territorial reading of space: state and pays. Basically, we assume that a State is a space of integration, whereas a pays is a space of attachment. The concept of pays was introduced in Cameroon from the metropolis with a meaning close to the concept of state, and it has been re-utilized by Cameroonian people depending on the successive contexts of colonization, decolonization, independence and, nowadays, democracy.
\end{abstract}

Key words: Colonisation, decolonisation, "pays", State, Cameroun

Resumo: ALGUMAS REFLEXÕES SOBRE A INTRODUÇÃO E A VULGARIZAÇÃO DO CONCEITO DE "PAYS" NOS CAMARÕES - Entre os conceitos cuja utilização indica a existência de uma leitura territorial do espaço estão os de Estado e "pays". Partimos do princípio de que o Estado é um espaço de integração (rattachement), ao contrário de "pays" que é um espaço de ligação (attachement). O conceito de "pays" foi introduzido nos Camarões com uma conotação mais próxima da de Estado, mas foi sujeito a uma reinterpretação pelos habitantes dos Camarões, no contexto dos sucessivos processos de colonização, descolonização, independência e, agora, democratização.

Palavras-chave: Colonização, descolonização, "pays”, Estado, Camarões.

1 Chercheur. Institut National de Cartographie, B.P. 14704 Yaoundé (Cameroun). Tel.: 237212922; Fax: 237201854; E-mail: bopda-at@ camnet.cm 
La réflexion qui suit est partie d'un constat: la pensée géographique commence quand l'espace sert de fond à une ou à des logiques de territoire. Or, parmi les concepts dont l'usage indique l'existence d'une lecture territoriale de l'espace, il en est deux qui reviennent très régulièrement en français: l'Etat et le pays. Mailles spatiales de référence des actions et des représentations, Etats et pays recouvrent la surface connue de la terre de leurs trames qui tantôt coïncident et tantôt diffèrent. En effet, dans sa logique géographique, l'Etat agit au niveau global comme un cadre institué pour empêcher la désagrégation d'un ensemble considéré comme un tout ou devant tenir en tant que tel, tandis que le pays agit comme une aspiration au niveau de chaque individu et suscite une attitude de recherche de l'agrégation en un ensemble que ces individus tiennent à voir émerger ou rester en un tout.

Nous partons du postulat de base que l'Etat est un territoire de rattachement, à la différence du pays qui est plutôt un territoire d'attachement. A l'Etat, fort de ses frontières qui le posent et l'imposent, s'oppose le pays fort de son milieu institué en repère fascinant. Pour caricaturer, on pourrait dire que l'Etat tient d'autorité et le pays par charisme! C'est avec cette trame théorique en fond que nous avons essayé de décrire les tribulations de la notion de pays dans la diffusion de son usage, de la France, métropole coloniale, au Cameroun, territoire d'outre-mer français, devenu aujourd'hui un Etat africain souverain.

\section{LE CONTEXTE CAMEROUNAIS}

Introduit au Cameroun il y a moins d'un siècle par la colonisation française, le mot pays y est, aujourd'hui, un des concepts géographiques les plus utilisés. Certes, les missionnaires anglais ou allemands ainsi que les explorateurs européens découvrant cet hinterland de la baie de Biafra avaient déjà institué pour leur propre gouverne un découpage de l'espace camerounais en territoires dédiés à différents groupes de populations supposées y vivre à demeure. Mais, en anglais ou en allemand, il n'y a pas d'équivalent exact du concept de "pays". Si le mot "country" est vite passé dans le pidjin-English avec "coundray", le sens que les nationaux en retiennent est surtout celui de "village" tel qu'il se retrouve dans l'expression très péjorative "come from coundray" pour dire "qui vient de la brousse" ou du village, le villageois étant alors synonyme d'ignorance et de "manque d'éducation".

Très bref, le passage des Allemands n'aura pas légué, en une quinzaine d'années, de mots particuliers rendant avec la même force ce que rend en français le concept de "pays". Entre 1889 et 1897, les Allemands localisent pourtant avec intérêt et précision autant les richesses naturelles dignes d'une exploitation que les groupes de populations vivant dans leur colonie. Par exemple, dans la carte de Yaoundé et ses environs à la fin du XIX ${ }^{\mathrm{e}}$ siècle, Zinker mentionne des noms de personnalités autochtones importantes ainsi que des groupes humains tels qu'ils se sont déclarés ou ont été déclarés. Pour les Allemands, il n'y a pas de pays, mais une lointaine colonie de l'empire perdue par son enclavement.

Ce sont donc les administrations suivantes et en particulier l'administration fran- 
çaise restée presque un demi-siècle au commandement de la partie orientale (la plus vaste) du pays qui, par leurs pratiques et l'enseignement scolaire, introduiront et vulgariseront l'usage du mot pays dans le Cameroun francophone. Pays signifie alors pour elles le Cameroun tout entier. La vision des indigènes est pourtant bien différente. Faute d'un vocable pour dire pays, dans leurs langues, ils vont systématiquement identifier les populations et des sites en disant simplement "chez qui" on est. Donné comme nom par les porteurs venant de la côte, Yaoundé signifie "Ya Ewondo", c'est-à-dire, chez les Ewondo.

Pour les indigènes, le vocable pays évoquera plutôt autre chose que l'ensemble d'un territoire colonial dont ils ne connaissent pour la plupart que très peu de chose, sinon "leur propre coin". Le pays, dans leur sens, c'est alors la terre natale, le territoire fondateur de l'ethnie et le village. L'école va jouer un rôle déterminant dans la diffusion de la langue française et de l'usage du mot pays. Les manuels scolaires élaborés par "les Blancs" pour "les Noirs", vont créer ou diffuser des images de pays. Il y a le pays du cours de géographie et d'histoire, tantôt milieu naturel, tantôt territoire supposé légitime de groupes sociaux dont celui, émergent, des Africains "occidentalisés" s'invente ou se découvre au jour le jour.

Fortement sollicité dès la fin des années 80 avec l'avènement du multipartisme, le pays local va resurgir sournoisement à la fin des années 90 pour se recycler dans la création de régions et de pouvoirs décentralisés. La constitution est renouvelée et le débat public voit s'introduire les concepts d'autochtones, d'allogènes. A peine les nouvelles générations, plus lettrées, ont-elles repris pour leur compte le Cameroun global comme pays qu'elles se trouvent piégées à leur tour par un débat sur les "pays" tels qu'ils devraient être. Le réajustement entre pays global et pays local devient une œuvre de Sysiphe.

\section{LA DIFFUSION COLONIALE INITIALE}

Colonie allemande placée sous le mandat puis sous la tutelle de la France et de l'Angleterre dès la fin de la guerre de 1914, le territoire du Cameroun n'a pris sa forme actuelle qu'au début du $\mathrm{XX}^{\mathrm{e}}$ siècle. L'apparition de l'Etat moderne n'y est donc pas le fait d'une dynamique endogène. Ce sont les Européens qui, au sortir de la conférence de Berlin en 1885, se partagent l'Afrique et fixent les principes de son découpage en colonies. Pour le Cameroun situé au fond du Golfe de Guinée, quelques explorations vont donner aux partisans de l'aventure impériale allemande en Afrique une idée préliminaire de la carte des peuples et des richesses de l'Hinterland. Pays est un mot français. En allemand, le terme "Land" n'en est pas vraiment l'équivalent et "Heimat" est plus proche de patrie. Les explorateurs comme les militaires ou les administrateurs allemands n'utiliseront pas, à proprement parler, d'équivalent de la notion de pays, pour désigner couramment soit leur colonie, soit les espaces occupés par les peuples indigènes d'Afrique qu'ils y trouvent souvent en mouvement ou en guerre au moment de leur installation.

$\mathrm{Si}$, par endroit ou par moment, ce sont des Africains, aristocrates ou non, qui ont 
appelé "les Blancs" pour se placer sous leur protection, cette installation européenne sur le continent va se heurter dans beaucoup de cas à des résistances de la part de certaines populations indigènes. Les résistants défendent ce qui constitue à leurs yeux leurs territoires, leurs peuples, leurs marchés et aussi, leurs "pays" au sens où ce terme s'utilise dans les langues locales. Accouché dans la douleur des "résistances africaines" de fin du XIX ${ }^{\mathrm{e}}$ siècle à l'invasion européenne, le territoire colonial d'outre-mer façonné à force de ruse, de coups de fusils, de canon et de baïonnette par les Allemands au cœur de l'Afrique centrale prendra le nom de Kamerun. Cette appellation étend celle de la cité côtière KamerunStadt (actuellement Douala) à partir de laquelle les Allemands, conformément aux termes des accords du congrès de Berlin de 1884-1885, organisent méthodiquement et résolument leur occupation de l'intérieur. Partagé comme un butin à la fin de la première guerre entre Français et Anglais vainqueurs des Allemands, le Kamerun verra sa partie orientale échoir à la France et par conséquent insérée dans l'univers de la francophonie où le vocable "pays" s'utilise.

Pour les Français recevant le Cameroun sous mandat de la Société des Nations (S.D.N.), c'est un pays au sens de territoire géopolitiquement reconnu comme individualisé au niveau international, avec un espace délimité et une population rattachée. Le mot pays passe d'autant mieux ici qu'il permet de désigner quelque chose qui n'est pas un Etat, du moins pas encore, mais qu'on ne peut traiter comme une partie du territoire proprement français. Le Cameroun est un pays au premier sens de ce mot qui signifie, selon le Petit Robert, dictionnaire français: "Territoire habité par une collectivité et constituant une réalité géographique dénommée". Faute d'Etat, on a le pays! Mais le pays camerounais global, tel que se le figurent les Européens pensant à un Etat, est luimême décrit en détail comme un composé de multiples pays divers. Il s'agit alors de pays au sens de région géographique plus ou moins nettement délimitée. La souplesse du concept de pays et sa polysémie en fait un concept mou qui s'emploie tant pour dire presque Etat, mais aussi pour désigner une portion d'espace considérée comme homogène dans son aspect physique ou socioculturel. La littérature coloniale présente alors avec une recherche parfois avouée du pittoresque un Cameroun aux multiples pays, dont les "pays de la forêt du sud", les "pays de la savane", les "pays Bamiléké et Bamoun".

Les populations africaines introduites dans l'univers de la francophonie vont adopter le concept de pays selon leur propre sensibilité. C'est ainsi que, arrivé de la métropole avec un sens plus proche de celui d'Etat, le concept de pays a connu une réinterprétation dans son utilisation par les Camerounais selon les contextes successifs de colonisation, de décolonisation, d'indépendance et, aujourd'hui, de démocratisation.

\section{LA RÉAPPROPRIATION CAMEROUNAISE DU CONCEPT DE PAYS}

Au Cameroun, dans un contexte de fraîche colonisation, l'usage fait du mot pays est double. Si pour beaucoup d'administrateurs ou d'Européens il est plus courant de parler $\mathrm{du}$ Cameroun dans son ensemble en tant que pays, les populations indigènes vont intérioriser cette façon de voir plutôt lentement. Pour la grande majorité des indigènes 
encore peu alphabétisés dans la langue française, le recours au concept de pays n'est même pas de mise. Pour la petite minorité des personnes instruites sur place, la tendance la plus courante est de parler de pays surtout pour évoquer d'abord leur terroir ou village d'origine, par opposition soit à la ville, soit au reste du Cameroun. Pour les plus lettrés, on voit de plus en plus apparaître une utilisation du terme dans deux sens. "Je vais au pays" déclare un Camerounais vivant en France qui décide de se rendre au Cameroun. Une fois au Cameroun, où il arrive soit par Douala, soit par Yaoundé, on l'entendra encore dire "je vais au pays" pour signifier qu'il veut se rendre dans son village d'origine ou dans la localité qui en tient lieu. Le pays se décline ainsi à deux échelles emboitées, exigeant chaque fois un recours au contexte de son évocation pour celui qui veut savoir de quoi on parle exactement quand on l'évoque. Cette situation prévaudra jusqu'à l'irruption du mouvement nationaliste indépendantiste de l'après-guerre.

Puis, dans un contexte de décolonisation qui commence avec les années 50, le recours au concept de pays comme ensemble du Cameroun connait une revalorisation. Dans le discours des nationalistes engagés qui vont aller jusqu'au maquis, il s'agit de "libérer le pays" divisé entre les Anglais et les Français, qui assurent sur lui une domination coloniale, et de le réunifier. Pour ceux qui hésitent à prendre l'option pour la décolonisation, "le pays" n'est pas encore assez mûr pour assurer son autonomie. L'enthousiasme ressenti lors de ce tournant historique pour une considération du pays au sens d'une nation entière et souveraine fera de cet autre sens du mot celui par lequel l'expression de l'émergence d'un Etat camerounais moderne rentre laborieusement dans l'histoire. En janvier 1955, Louis Paul Aujoulat, un des acteurs français les plus déterminants de la période de la lutte pour l'indépendance, considère par exemple que les partisans camerounais de l'Union des Populations du Cameroun, le mouvement nationaliste le plus engagé, ne se préoccupent pas vraiment de "préparer solidement le bonheur de leur pays". Le choix en quelque sorte posé aux populations indigènes est celui de savoir si elles vont adopter le sens global et proto-étatique donné au mot pays par le colonisateur ou se replier sur celui plus restrictif que leur attachement aux terroirs ancestraux leur impose.

L'attachement aux pays précoloniaux tribaux reste fort, alors que le rattachement au Cameroun, pays moderne, se fait fort. Ce double attachement au territoire de rattachement colonial d'hier et aux territoires d'attachement "traditionnels" d'avant la colonisation va pousser les élites de la fin de la période coloniale à composer un hymne national où, sans paraître directement, l'idée de la double appartenance aux pays villageois et au pays camerounais est magnifié en ces termes dans le titre même de l'hymne national: "Au Cameroun berceau de nos ancêtres". L'indépendance acquise dans le sang s'est traduite concrètement par une nationalisation de la haute hiérarchie de l'administration, offrant ainsi un nouveau contexte à l'utilisation du mot "pays". Nous avons désigné ce contexte d'“années Ahidjo", du nom du premier président de la république du Cameroun arrivé au pouvoir en 1957 et parti en 1982.

Dans un contexte de nationalisation de la haute hiérarchie de l'administration que l'indépendance a ouvert avec l'ère Ahidjo, le recours au concept de pays n'est plus accepté et acceptable que comme pensée unique de la "nation unie", qui se fait fort de 
se doter d'un "pays prospère". "L'unité nationale" est devenue, pendant près d'un quart de siècle, l'idéologie centrale de l'édification du "pays". Quand l'heure est au nationalisme ou au patriotisme activiste, c'est que, dans les consciences en présence, l'ajustement du pays - niveau géographique d'attachement - à l'Etat - niveau géographique de rattachement - est en question.

\section{LA PÉRIPÉTIE DÉMOCRATIQUE ET MULTIPARTISANNE ACTUELLE}

Près d'un demi-siècle de discours unique fort sur l'unité nationale pendant "l'ère Ahidjo" semblait avoir, de 1960 à 1982, stabilisé une vision du pays essentiellement instituée dans les sphères du discours officiel comme une entité camerounaise unie et totale. A la base, les populations continuaient certes à parler des pays dans le pays pour indiquer leur village d'origine, mais l'impression donnée était celle qu'il ne s'agissait bien là que de quelques formes résiduelles d'une pratique condamnée à disparaître. La menace des pays locaux sur le pays global semblait subjuguée. Du début au milieu de cette période, des remous régionalistes ou tribalistes importants furent enregistrés. Mais la force de l'attachement au pays global camerounais semblait tenir face aux tentatives de "subversion" des attachements aux pays locaux du Cameroun, lorsque surviennent les secousses de la libéralisation politique de la fin des années 80 avec "l'ère Biya" (du nom du président actuel).

En imposant aux élites du pays de se redistribuer le marché politique sur la base de la concurrence ouverte entre des partis sans limitation de nombre autre que la sanction des urnes, l'instauration de la démocratie à la fin des années 80 allait déclencher une évolution significative de la notion de pays ou des réactions qui en relèvent. Côté cour, tous les leaders en quête de soutien populaire ont proclamé que leurs actions se situaient dans la stricte défense des intérêts bien compris du "pays" global. Côté jardin, il s'en est trouvé, nombreux, à manœuvrer en tirant sur la corde régionaliste ou sectaire, indiquant clairement leur aspiration à se réserver exclusivement leur pays local (au sens de pays d'origine) dans le pays global. La dynamique interactive de l'exclusion entre les pays locaux menace la survie même du pays global qu'est l'Etat camerounais.

Exacerbé par la situation de crise économique mettant à mal le pouvoir d'achat des adultes et l'accès des jeunes à l'emploi de bureau après une scolarisation réussie, l'agacement d'énormes couches de population à ne pouvoir réussir leur vie comme elles y aspiraient a contribué à un repli sur le "pays d'origine" supposé, à tort ou à raison, comme le seul refuge encore possible pour faire face aux difficultés socioéconomiques. La carte des zones d'influence ou d'action des partis politiques dans le contexte du nouveau multipartisme s'est trouvée également piégée par le tropisme des mailles floues mais fortes des pays locaux, et le pays global camerounais en a bien souffert. Certaines de ces mailles sont anciennes et existaient déjà dans les années 60 au moment de l'indépendance. D'autres n'ont pris leur importance qu'avec les circonstances particulières de l'irruption de la démocratie et du multipartisme en Afrique à la fin des années 80. Des oppositions supposées ou réelles sont entrées comme des traditions dans les discours politiques ou de politique: 
- opposition de blocs historiques entre les pays locaux anglophones et les pays locaux francophones;

- opposition régionale ou régionaliste entre un nord et un sud;

- opposition économique entre "les pays" enclavés et "les autres pays";

- opposition socio-politique ou culturelle entre pays locaux de "minorités" et pays locaux de "majorités";

- au sein des mêmes localités, l'esprit de terroir plus ou moins réactivé aux forceps par des "élites locales intérieures et extérieures" s'est fait fort de ressortir des distinctions entre "autochtones" et "allogènes".

Dans les confrontations qui s'en sont suivies, la force de borne du chaos du pays global camerounais a souffert sans rompre, et celle de centre d'attache que sont les pays locaux s'est réactivée sans véritablement primer sur la première. L'interdépendance des composantes a montré sa fragilité et, en même temps, sa nécessité.

Aujourd'hui, l'ajustement entre "pays global" et "pays locaux" est tout autant d'actualité qu'il y a un siècle environ, quand il fallut bien que des peuples jadis séparés ou distinct acceptent de vivre ensemble dans un même "pays" sous la contrainte ou le contrôle des métropoles impérialistes européennes imposant leur "paix coloniale".

\section{CONCLUSION}

Récupéré du vocabulaire des expatriés européens séjournant en Afrique par des nationaux africains s'ouvrant au monde moderne, le mot pays est un concept des plus mous qui soit. Pays est utilisé au sens de contrée, endroit, région. Employé à la fois pour désigner le territoire habité par une collectivité et les gens ayant ce territoire comme terre d'élection, le mot pays prend aussi le sens de patrie. En ce sens, il finit par évoquer en quelque sorte le territoire d'attachement, par opposition au territoire de rattachement qu'évoquerait plus l'Etat. Le succès de la diffusion du concept vient finalement du fait qu'il permet à lui tout seul d'exprimer plusieurs choses parfois bien différentes sans avoir l'air de changer vraiment de sujet, alors que les sous-entendus des discours en présence concernent des choses aussi délicates que l'attachement patriotique ou la légitimité dans la citoyenneté. Le flou et le mou du concept de pays lui donnent une capacité de pénétration étonnante.

Véritable chambre à écho des tensions de l'intégration sociale, politique, voire géopolitique, dans la société africaine ou européenne, l'invention ou la gestion du sens donné au mot "pays" et les chevauchements, les changements de géométrie ou d'échelle que ce qu'il recouvre a subi, sont autant de révélateurs ou d'indicateurs des tribulations du sentiment de citoyenneté multi-scalaire et poly-centrée vécu par les populations concernées ou par leurs élites. On ne peut finalement bien comprendre la circulation d'un tel concept autour du monde, ici le monde francophone, qu'en se penchant en même temps sur l'émergence et la pérennité de communautés scientifiques, administratives ou politiques qui se sont simultanément mises en place en faisant du recours à ce concept leur fond de commerce. Dans tous les cas, les pays ont toujours 
une histoire, une histoire toujours inachevée. Celle-ci restitue les regards toujours croisés de ceux qui s'y intéressent ou de ceux qui recourent à l'utilisation de l'idée que ce mot recouvre: l'histoire de l'arbre et de la pirogue, de l'enracinement et de la mobilité, de la constance et du changement. 